\title{
Dielectric Anisotropy of the GaP/Si(001) Interface from First-Principles Theory
}

\author{
Pankaj Kumar and Charles H. Patterson* \\ School of Physics, Trinity College Dublin, Dublin 2, Ireland \\ (Received 15 February 2017; published 9 June 2017)
}

\begin{abstract}
First-principles calculations of the dielectric anisotropy of the $\mathrm{GaP} / \mathrm{Si}(001)$ interface are compared to the anisotropy extracted from reflectance measurements on $\mathrm{GaP}$ thin films on $\mathrm{Si}(001)$ [O. Supplie et al., Phys. Rev. B 86, 035308 (2012)]. Optical excitations from two states localized in several Si layers adjacent to the interface result in the observed anisotropy of the interface. The calculations show excellent agreement with experiment only for a gapped interface with a P layer in contact with Si and show that a combination of theory and experiment can reveal localized electronic states and the atomic structure at buried interfaces.
\end{abstract}

DOI: 10.1103/PhysRevLett.118.237403

Thin layers of III-V semiconductors grown on $\mathrm{Si}(001)$ have been widely investigated for III-V on-silicon integration [1]. Applications for $\mathrm{GaP}$ on $\mathrm{Si}$ include buffer layers in multijunction $\mathrm{GaAsP} / \mathrm{Si}$ photovoltaics [2,3]. Obtaining a high quality interface and avoiding defects such as threading dislocations [3] or antiphase domains $[4,5]$ in the GaP layer is essential to the performance of III-V optoelectronic devices [3]. Techniques for observing the microscopic properties of the buried interface between a thin dielectric layer and an underlying dielectric substrate are limited. The short mean free paths of charged particles in matter, lack of interface sensitivity, and need for ultrahigh vacuum mean that electron spectroscopies are unsuitable. On the other hand, light has a great potential for directly probing buried interfaces since visible light can penetrate thin layers and reflect from the interface.

An optical probe can have interface (or surface) sensitivity when the reflecting boundary is anisotropic and the surrounding bulk media are isotropic. The (001) interfaces between tetrahedral semiconductors are anisotropic while bulk tetrahedral semiconductors are isotropic on a mesoscopic length scale. Light induces transitions between valence and conduction band states in the bulk and at an interface, but only transitions between states localized near the interface contribute to the difference in reflectance parallel and perpendicular to rows of atoms at the interface. This is known as reflectance anisotropy (RA) and the associated difference in dielectric functions is the interface dielectric anisotropy (IDA).

In this Letter we report first-principles calculations of the IDA of thin GaP layers on $\mathrm{Si}(001)$ and compare them to experimental measurements by Supplie et al. [5]. The agreement between theory and experiment is excellent when the last complete layer in $\mathrm{GaP}$ is $\mathrm{P}$ (as in the experiment) and when the interface is "gapped," i.e., when there is a gap between the interface valence and conduction band states. A modified version of the electron counting arguments by Pashley [6] given in the
Supplemental Material [7] shows that when the GaP surface is terminated by $\mathrm{P}$ dimers with one $\mathrm{H}$ atom per dimer, there is one electron more per dimer than is needed to satisfy valence requirements at the surface. This electron transfers to the $\mathrm{GaP} / \mathrm{Si}$ interface and a gapped interface results when the $\mathrm{Si}$ layer is doped with one $\mathrm{Ga}(\mathrm{P})$ per surface dimer, when the last $\mathrm{GaP}$ layer is $\mathrm{P}(\mathrm{Ga})$. Electrons transferred to the interface occupy localized states at the buried interface.

Below we show that it is the optical transitions between these interface localized states that are responsible for the observed IDA [5]. The states are localized within three Si bilayers of the interface. Their filling determines whether the interface is electrically conducting or semiconducting. The predicted IDA is strikingly different when the last layer is $\mathrm{Ga}$ or $\mathrm{P}$ and when the interface is electrically conducting. Measurement of the IDA at buried interfaces interpreted by first-principles calculations therefore offers a unique means of probing the atomic and electronic states of interfaces between dielectrics. The approach that is applied here was previously applied to the RA of surfaces of systems including clean $\mathrm{Si}$ and $\mathrm{Ge}(001)$ [8] and clean and transition metal covered $\mathrm{Si}(111)$ [9-11]. As far as the authors are aware, this is the first application of first-principles calculations to determine the semiconductor buried interface anisotropic optical response.

RA [5,12,13], LEED, XPS, and density functional theory (DFT) calculations $[12,14]$ have shown that GaP grown on $\mathrm{Si}(001)$ by MOVPE has a mixed $(2 \times 2)$ and $c(4 \times 2) \mathrm{P}$ dimer surface. The dimers are tilted and have a $\mathrm{H}$ atom on the down-tilted $\mathrm{P}$ atom. This is supported by DFT calculations of the surface energies of $\mathrm{GaP}(001)$ surfaces as a function of the $\mathrm{P}$ and $\mathrm{H}$ chemical potential [15], which show that the $\mathrm{GaP}(001)-(2 \times 2) \mathrm{H}$ monohydride surface has the lowest formation energy at moderate $\mathrm{P}$ and $\mathrm{H}$ chemical potentials.

Interface RA has been measured in several cases including $\mathrm{InP} / \mathrm{GaAs}(001)$ [16], $\mathrm{ZnSe} / \mathrm{GaAs}(001)$ and 


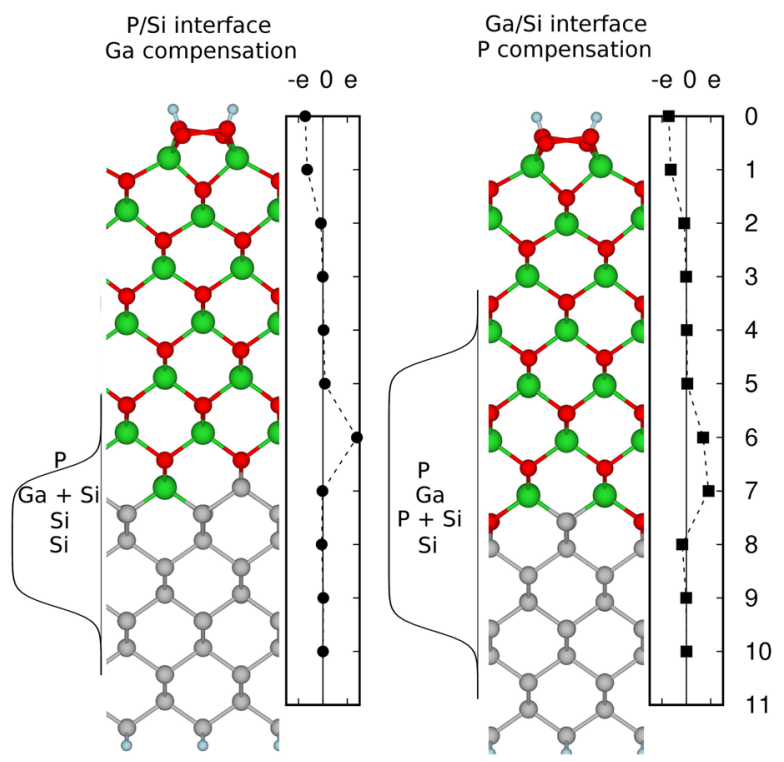

FIG. 1. Atomic structures of slabs with $(2 \times 2) \mathrm{H}$ surface terminations used for the IDA calculations. Left: GaP layer terminated in a $\mathrm{P} / \mathrm{Si}$ interface with $\mathrm{Ga}_{\mathrm{Si}}$ substitution in the first $\mathrm{Si}$ layer. The net charges in Ga-P or Si bilayers are shown in the panels on the right of the slabs. The filter functions used to extract contributions to the dielectric function from specific layers are shown on the left of the slabs for Si bilayers adjacent to the GaP thin film and for bilayers on both sides of the interface. Right: $\mathrm{GaP}$ layer terminated in a $\mathrm{Ga} / \mathrm{Si}$ interface with $\mathrm{P}_{\mathrm{Si}}$ substitution in the first $\mathrm{Si}$ layer. The small spheres are $\mathrm{H}$ atoms, the medium sized red spheres are $\mathrm{P}$ atoms, the large green spheres are $\mathrm{Ga}$ atoms, and the medium sized gray spheres are Si atoms.

$\mathrm{SiO}_{2} / \mathrm{Si}(112)$ [17], $\mathrm{AlAs} / \mathrm{GaAs}(001)$ [18], and $\mathrm{GaP} /$ $\operatorname{Si}(001)[5,12,13]$. There have been few calculations of optical interface anisotropies. Optical anisotropies of $\mathrm{ZnSe} / \mathrm{GaAs}(001)$ [19] and $\mathrm{AlAs} / \mathrm{GaAs}(001)$ [18] interfaces have been calculated using tight-binding electronic structure methods.

The calculation of the RA,

$$
\frac{\Delta r}{r}=2 \frac{r_{x}-r_{y}}{r_{x}+r_{y}}
$$

where $r_{\alpha}(\alpha=x, y)$ is the complex reflection coefficient along a particular axis, is usually performed for surfaces using dielectric functions for the bulk material and a slab terminated by the surface reconstruction of interest. These are used in a three-layer model proposed by McIntyre and Aspnes [20]. Yasuda [21] proposed a five-layer model (ambient, thin surface layer, thin film, thin interface layer, and bulk medium, Fig. 1) to account for the RA of a thin film, of non-negligible thickness compared to the wavelength of light, $\lambda$, on a bulk medium. It was developed further by Hunderi et al. [18] in 2005. According to this version of the five-layer model, the RA can be expressed as $[5,18]$

$$
\frac{\Delta r}{r}=a\left(d_{2}, \lambda\right) d_{1} \Delta \epsilon_{1}+b\left(d_{2}, \lambda\right) d_{3} \Delta \epsilon_{3},
$$

where

$$
\begin{aligned}
a\left(d_{2}, \lambda\right) & =\frac{4 \pi i}{\lambda} \frac{n_{0}}{\epsilon_{0}-\epsilon_{2}}\left(1-\frac{n_{2}}{n_{0}} c\left(d_{2}, \lambda\right)\right), \\
b\left(d_{2}, \lambda\right) & =\frac{4 \pi i}{\lambda} \frac{n_{2}}{\epsilon_{2}-\epsilon_{4}} c\left(d_{2}, \lambda\right), \\
c\left(d_{2}, \lambda\right) & =r_{24} \frac{1-r_{02}^{2}}{\left(r_{02}+r_{24} e^{2 i \beta_{2}}\right)\left(1+r_{02} r_{24} e^{2 i \beta_{2}}\right)}, \\
\Delta \epsilon_{i} & =\epsilon_{x x, i}-\epsilon_{y y, i} .
\end{aligned}
$$

$r_{i j}$ is the complex reflection coefficient for the interface between thick phases $i$ and $j$ (in this case, $r_{02}$ for the ambient and GaP thin film, and $r_{24}$ for the GaP thin film and bulk $\mathrm{Si}$ ) and $\beta_{i}=2 \pi n_{i} d_{i} / \lambda$ is a phase acquired by propagation through layer $i$, of thickness $d_{i}$ and complex refractive index $n_{i}$. Multiple reflections at boundaries of the thin surface and interface layers are included in these coefficients. $d_{1} \Delta \epsilon_{1}$ and $d_{3} \Delta \epsilon_{3}$ are the surface dielectric anisotropy and IDA, respectively. In this work, the P dimer bonds are aligned with the $x$ direction.

According to Eq. (2), differences in the reflection coefficient for either polarization of light in normal incidence depend on factors that contain only optical constants of thick layers $(i=0,2$, and 4$)$ and an overlying film thickness $d_{2}$ times differences in the dielectric functions of the thin layers [5]. Using pairs of RA measurements from a series of $\mathrm{GaP}$ thin films on $\mathrm{Si}(001)$ of differing thickness, Supplie et al. extracted the surface dielectric anisotropy and IDA and the functions $a$ and $b$ in Eq. (2) $[5,13]$. These extracted dielectric anisotropies are compared to the results of hybrid DFT calculations presented below.

A GaP layer grown on an atomically flat $\mathrm{Si}(001)$ surface with a $(2 \times 2) \mathrm{H}$ surface reconstruction above an integer number of Ga-P bilayers has one electron more per surface dimer than is needed for a saturated bond network (see the Supplemental Material [7]). Excess electrons from surface dimers could occupy localized states at the surface. Alternatively, they could compensate holes created by replacing one $\mathrm{Si}$ atom per dimer in the Si layer by Ga. The valence band edge of $\mathrm{Si}(001)$ in contact with $\mathrm{GaP}$ lies $0.5 \mathrm{eV}$ above the $\mathrm{GaP}$ valence band edge in our calculations, making the Si layers adjacent to the interface a low energy reservoir for holes. The transfer of two electrons per $(2 \times 2)$ cell from the GaP surface to the interface in order to satisfy valence electron counting would generate an interface charge density of $-0.5 \mathrm{Cm}^{-2}$ and an electric field strength of $2.9 \times 10^{10} \mathrm{~V} \mathrm{~m}^{-1}$ outside the interface charge layer. A field of this magnitude generates a surface charge density of $-2.6 \mathrm{C} \mathrm{m}^{-2}$ via linear response, assuming a susceptibility of $\chi=10$ for the $\mathrm{GaP}$ thin layer. According to our hybrid DFT calculations, the surface does indeed become negatively charged, but to a lesser degree $\left(-0.4 \mathrm{C} \mathrm{m}^{-2}\right)$. The net negative charge on the $\mathrm{P}$ and $\mathrm{H}$ dimer layer [corresponding to about $-1.5 e$ per $(2 \times 2)$ cell] 


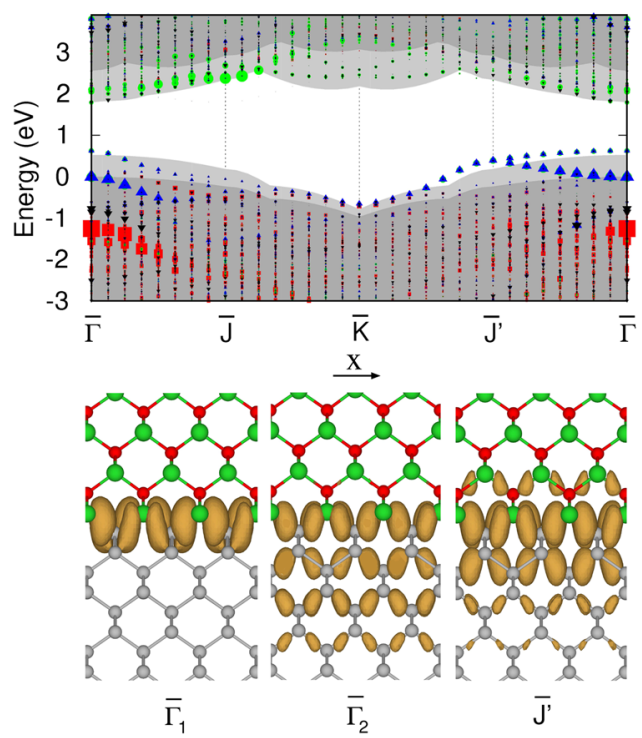

FIG. 2. Atom projected band structure of the $\mathrm{P} / \mathrm{Si}$ interface with Ga compensation (upper panel). The atom layers onto which bands are projected correspond to the four layers marked on the left of Fig. 1. P layer, red squares; mixed $\mathrm{Ga} / \mathrm{Si}$ layer, green circles; second Si layer, blue upward triangles; third Si layer, black downward triangles. Bulk band projections for $\mathrm{GaP}$ and $\mathrm{Si}$ are shown as dark and light shading, respectively. The GaP bulk valence band maximum is the reference energy. Modulus squared of interface localized states at $\bar{\Gamma}$ and $\bar{J}^{\prime}$ points of the Brillouin zone (lower panel).

and on each Ga-P bilayer and several Si bilayers at gapped $\mathrm{P} / \mathrm{Si}$ and $\mathrm{Ga} / \mathrm{Si}$ interfaces is shown in Fig. 1. Interfaces have a corresponding net positive charge, mainly from depletion of charge on the Ga ions, which results in layer expansion at the interface. Replacement of a P layer by Si to form a sharp $\mathrm{Ga} / \mathrm{Si}$ interface creates four holes at the interface, which are filled by the transfer of two excess electrons per $(2 \times 2)$ cell from the surface and replacing two atoms in the Si layer by P. Hence, a gapped $\mathrm{Ga} / \mathrm{Si}$ interface also requires the transfer of two electrons from the surface to the interface. The net charge at gapped $\mathrm{P} / \mathrm{Si}$ and $\mathrm{Ga} / \mathrm{Si}$ interfaces is the same, but the charge distribution over bilayers (Fig. 1) and their IDAs differ. The charge on each bilayer at either surface is essentially identical while the lower, pseudo- $\mathrm{H}$ atom terminated $\mathrm{Si}$ surface carries close to zero net charge.

The electronic structure of the slab used for hybrid DFT calculations, projected onto four atomic layers at a gapped $\mathrm{P} / \mathrm{Si}$ interface, is shown in Fig. 2. There are two occupied interface localized states, which lie above the GaP layer valence band maximum throughout most of the Brillouin zone. The moduli squared for these states at the $\bar{\Gamma}$ and $\bar{J}^{\prime}$ points of the Brillouin zone are also shown in Fig. 2. The lower energy interface state at $\bar{\Gamma}$ (labeled $\bar{\Gamma}_{1}$ ) is strongly localized in the Si layer, which contains two Ga dopants and the Si bilayer immediately beneath. The higher energy interface state at $\bar{\Gamma}$ (labeled $\bar{\Gamma}_{2}$ ) extends two Si bilayers further from the interface. At $\bar{J}^{\prime}$ where the interface states are quasidegenerate, there is an intermediate degree of localization and the state penetrates some way into the GaP layer.

Two interface states with similar localization and dispersion as those just described for the gapped $\mathrm{P} / \mathrm{Si}$ interface are found at the gapped $\mathrm{Ga} / \mathrm{Si}$ interface (see Fig. 4 of the Supplemental Material [7]). Compensated P/Si and $\mathrm{Ga} / \mathrm{Si}$ interfaces have two $\mathrm{Si}$ atoms replaced by either $\mathrm{Ga}$ or P. Further calculations were performed in which these atoms were successively replaced by $\mathrm{Si}$, leaving one or two electrons $(\mathrm{P} / \mathrm{Si})$ or holes $(\mathrm{Ga} / \mathrm{Si})$ in either slab. Electrons in the $\mathrm{P} / \mathrm{Si}$ slab become localized on atoms in the layer that contained compensating $\mathrm{Ga}$ atoms. A band becomes half filled when both Ga's are replaced by Si to yield an abrupt $\mathrm{P} / \mathrm{Si}$ interface and the Fermi level shifts above $1 \mathrm{eV}$ above the $\mathrm{GaP}$ valence band maximum. Holes in the slab with the $\mathrm{Ga} / \mathrm{Si}$ interface localize in the interface states in the $\mathrm{Si}$ layers described above.

The CRYSTAL program [22] was used to obtain the relaxed atomic coordinates of slabs representing the $\mathrm{GaP} / \mathrm{Si}(001)$ surfaces and interfaces and their electronic structures. Dielectric functions were calculated using wave function and energy band structures from CRYSTAL as input to the Exciton code [23]. See the Supplemental Material [7] for details of the basis sets, the pseudopotentials, and the computational parameters used in this work, or the original papers in Refs. [24-30]. The hybrid DFT functional [31] modified for the calculations of surface and interface dielectric anisotropies in this work has been described elsewhere [8]. The expression used to calculate the dielectric functions in Eq. (2) contains the electron momentum operator, which is expressed in a local orbital basis. A filter function is applied to the momentum operator matrix elements, which selects contributions to the slab dielectric functions from the initial state wave functions in the surface or interface regions. Similar approaches have been applied previously for this purpose [32-34] and the method used here is described in the Supplemental Material [7]. The overall effect of these filters (Fig. 1) is to turn off contributions to the dielectric functions from parts of the initial state wave functions that lie outside them.

The imaginary parts of the interface dielectric functions, $\operatorname{Im}\left(\epsilon_{x x, 3}\right)$ or $\operatorname{Im}\left(\epsilon_{y y, 3}\right)$, times the interface layer thickness $d_{3}$ for normal incidence radiation are shown in the top panels of Fig. 3. $\epsilon_{x x, 3}$ is calculated with the electric vector polarized parallel to the surface dimers (and atomic rows in the lowest complete Ga-P bilayer). $\epsilon_{y y, 3}$ is calculated with the electric vector polarized perpendicular to the dimers. The IDA is the difference in these functions, $d_{3} \Delta \epsilon_{3}=d_{3}\left(\epsilon_{x x, 3}-\epsilon_{y y, 3}\right)$. The interface dielectric functions and the IDA for the gapped $\mathrm{P} / \mathrm{Si}$ and $\mathrm{Ga} / \mathrm{Si}$ interfaces are shown in the lower panels of Fig. 3. Theoretical data are shown up to $6 \mathrm{eV}$ as there is a major dip above $4 \mathrm{eV}$ that could be measured in future experimental work. The IDA functions for both types of interface show similar curves, with a crossover from a positive to negative IDA above 


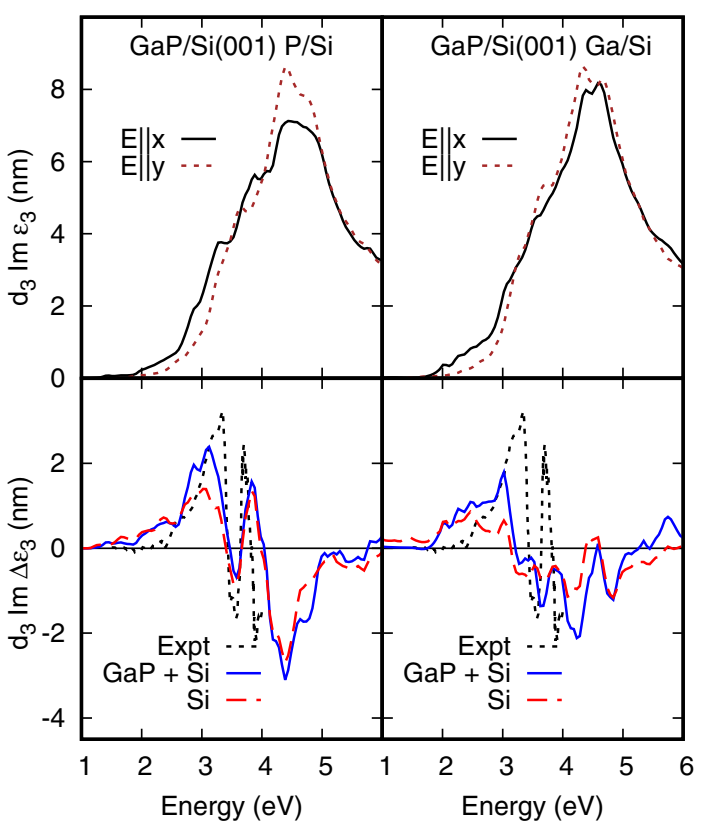

FIG. 3. Upper panel: interface dielectric functions of gapped $\mathrm{P} / \mathrm{Si}$ and $\mathrm{Ga} / \mathrm{Si}$ interfaces with the incident radiation electric vector aligned parallel or perpendicular to the $\mathrm{P}$ dimer bond (solid and dotted curves, respectively). Lower panel: the IDA calculated for gapped $\mathrm{P} / \mathrm{Si}$ and $\mathrm{Ga} / \mathrm{Si}$ interfaces using filter functions of width three $\mathrm{Si}+$ three $\mathrm{GaP}$ bilayers (solid curves), three $\mathrm{Si}$ bilayers (dashed curve), and experiment redrawn from Ref. [5].

$3 \mathrm{eV}$, but the agreement between the gapped $\mathrm{P} / \mathrm{Si}$ interface and experiment in the lower left panel is clearly much better. The first layer deposited in MOVPE of GaP on $\mathrm{Si}(001)$ was $\mathrm{P}$ in the IDA data shown [5].

In order to determine from which region the IDA originates, the IDA spectra were compared with the filter function arranged to capture the IDA contributions from both the $\mathrm{GaP}$ and $\mathrm{Si}$ sides of the interface and to eliminate any IDA contribution from GaP. Comparison to the experimental data (dotted line in Fig. 3) shows excellent agreement with experiment for the gapped $\mathrm{P} / \mathrm{Si}$ interface. There is little difference in the predicted IDA when the filter captures contributions from both sides of the interface compared to when only contributions from the Si side are included. Hence, optical transitions from states localized in Si layers near the interface cause the observed IDA. The shape of the positive peak below $3 \mathrm{eV}$ and the strong dip at $3.5 \mathrm{eV}$ are reproduced. In contrast, the positive peak for the gapped $\mathrm{Ga} / \mathrm{Si}$ interface is shifted to a lower energy than the experimental peak and the dip at $3.5 \mathrm{eV}$ and extra peak at $4.0 \mathrm{eV}$ are not reproduced. IDA functions for gapped interfaces and conducting interfaces with electrons or holes localized at interfaces are compared to experimental data in the Supplemental Material [7]. Conducting interfaces have IDAs that are not in agreement with experiment, showing that the interfaces reported by Supplie et al. were gapped [5].

Finally, optical transitions and occupied and unoccupied states that are responsible for the observed IDA were identified by inspecting the strongest transitions at 3.0 and $3.5 \mathrm{eV}$ (i.e., at the positive peak and the negative dip in the IDA spectrum). The strengths of optical transitions at a given photon energy $E$ with the electric field vector along the direction $\alpha$ are determined by the moduli squared of the momentum matrix elements and transition energies, $\left|p_{n n^{\prime} \mathbf{k}}^{\alpha}\right|^{2} \delta\left(E-E_{n n^{\prime} \mathbf{k}}\right) . n$ and $n^{\prime}$ are the initial and final state labels at $k$ point $\mathbf{k}$ and $E_{n n^{\prime} \mathbf{k}}$ is an interband transition energy. The strongest optical transitions at $3.0 \mathrm{eV}$ for a gapped $\mathrm{P} / \mathrm{Si}$ interface with the electric vector aligned with the $x$ direction occur between the initial states, which are the occupied interface states that are highest in energy, and vacant interface-localized states along the $\overline{\Gamma J}$ edge of the Brillouin zone (Fig. 2). The dip at $3.5 \mathrm{eV}$ arises from transitions from interface states with $k$ vectors lying along another edge of the Brillouin zone $\left(\overline{\Gamma J}^{\prime}\right)$ and the electric field aligned with the $y$ direction. These transitions occur at higher energy $(3.5 \mathrm{eV})$ because of the downward dispersion of the interface states along $\overline{\Gamma J}$. Transitions from interfacelocalized initial states in other parts of the Brillouin zone are much weaker. Hence, the dielectric anisotropy at the $\mathrm{GaP} / \mathrm{Si}(001)$ interface arises from localized states in $\mathrm{Si}$ bilayers adjacent to the $\mathrm{GaP}$ thin layer and the main contributions come from states along high symmetry directions in the Brillouin zone.

In summary, electron counting using conventional ideas about valence at the $\mathrm{GaP} / \mathrm{Si}(001)$ surface and interface shows that the number of electrons in the $\mathrm{P}$ dimer layer is too large by one per dimer. A gapped surface and interface is formed by replacing one Si per dimer by a Ga for a $\mathrm{P}$ terminated GaP layer or by replacing one Si per dimer by a $\mathrm{P}$ for a Ga terminated GaP layer. The transfer of electrons from the surface to the interface results in a large, negative areal charge density at the interface. The linear response of the GaP layer to the negative layer results in reversal of the net charge transfer and net negative (positive) surface (interface) layers. The resulting electric field across the GaP layer, predicted to be of order $2 \times 10^{10} \mathrm{~V} \mathrm{~m}^{-1}$, may help to dissociate bound electron-hole pairs in a photovoltaic cell. Electrons transferred to the interface are trapped within three $\mathrm{Si}$ bilayers closest to the $\mathrm{GaP}$ layer. The optical transitions of these trapped electrons at gapped $\mathrm{P} / \mathrm{Si}$ interfaces agree with the data; uncompensated, ungapped conducting interfaces, or gapped $\mathrm{Ga} / \mathrm{Si}$ interfaces, do not. A combination of optical measurement and theoretical calculation can therefore be used to determine the atomic structure at the interface and the gapped or ungapped conducting character of the interface electrons.

From the work presented here and previous experimental work [5], we expect that the IDA can be measured and calculated for technologically important (100) interfaces of tetrahedral semiconductors such as GaN [35] or AlN [36] on $\mathrm{Si}(100)$, yielding insight into their atomic and electronic structures. The IDA is a particular (normal incidence) case of spectroscopic ellipsometry. A combination of theory and experiment similar to that presented here could be used to determine the dependence of the interface valence and 
conduction band electronic structure on the interface atomic structure, exploiting the metallicity of the interface between insulating layers to isolate the interface contribution. The $\mathrm{LaAlO}_{3} / \mathrm{SrTiO}_{3}$ interface has a complex atomic structure, which may contain stripes of $\mathrm{O}$ vacancies [37] and has an intermixing of cations [38]. It has been used to construct a field effect transistor [39]. Ellipsometric measurements have been used to probe the electronic behavior and charge transfer at the interface [40,41]. A combination of spectroscopic ellipsometric measurements and theoretical calculations therefore has great potential as a probe of the electronic states of buried interfaces.

P. K. is grateful to Trinity College Dublin for studentship support. Calculations were performed on the Kelvin cluster, maintained by the Trinity Centre for High Performance Computing, funded through grants from the Irish Higher Education Authority through its PRTLI programme and the Fionn cluster, maintained by the Irish Centre for High End Computing and funded by Science Foundation Ireland. The authors wish to thank Thomas Hannappel and Oliver Supplie for discussions on their experimental data and John McGilp for a critical reading of the Letter.

*Corresponding author.

Charles.Patterson@tcd.ie

[1] H. Kroemer, J. Cryst. Growth 81, 193 (1987).

[2] T. J. Grassman, A. M. Carlin, J. Grandal, C. Ratcliff, L. Yang, M. J. Mills, and S.A. Ringel, Proc. SPIE 8256, Physics, Simulation, and Photonic Engineering of Photovoltaic Devices, 82560R (2012).

[3] J. R. Lang, J. Faucher, S. Tomasulo, K. N. Yaung, and M. L. Lee, Appl. Phys. Lett. 103, 092102 (2013).

[4] I. Németh, B. Kunert, W. Stoltz, and K. Volz, J. Cryst. Growth 310, 1595 (2008).

[5] O. Supplie, T. Hannappel, M. Pristovsek, and H. Döscher, Phys. Rev. B 86, 035308 (2012).

[6] M. D. Pashley, Phys. Rev. B 40, 10481 (1989).

[7] See Supplemental Material at http://link.aps.org/ supplemental/10.1103/PhysRevLett.118.237403 for electron counting used to account for the electronic structures found at compensated and uncompensated $\mathrm{P} / \mathrm{Si}$ and $\mathrm{Ga} / \mathrm{Si}$ interfaces in hybrid DFT calculations as well as the $\mathrm{GaP} / \mathrm{Si}(001)$ $(2 \times 2) \mathrm{H}$ surface; methods used to calculate surface and interface dielectric functions of slabs used in this work and details of electronic structure calculations are also given.

[8] S. Banerjee, J. F. McGilp, and C. H. Patterson, Phys. Status Solidi B 252, 78 (2015).

[9] C. H. Patterson, S. Banerjee, and J. F. McGilp, Phys. Rev. B 84, 155314 (2011).

[10] S. Jorgji, J. F. McGilp, and C. H. Patterson, Phys. Rev. B 87, 195304 (2013).

[11] C. H. Patterson, S. Banerjee, and J. F. McGilp, Phys. Rev. B 94, 165417 (2016).
[12] O. Supplie, S. Bruckner, O. Romanyuk, H. Doscher, C. Hohn, M. M. May, P. Kleinschmidt, F. Grosse, and T. Hannappel, Phys. Rev. B 90, 235301 (2014).

[13] O. Supplie, M. M. May, G. Steinbach, O. Romanyuk, F. Grosse, A. Naagelein, P. Kleinschmidt, S. Bruckner, and T. Hannappel, J. Phys. Chem. Lett. 6, 464 (2015).

[14] O. Romanyuk, O. Supplie, T. Susi, M. M. May, and T. Hannappel, Phys. Rev. B 94, 155309 (2016).

[15] P. H. Hahn, W. G. Schmidt, F. Bechstedt, O. Pulci, and R. DelSole, Phys. Rev. B 68, 033311 (2003).

[16] O. Acher, S. M. Koch, F. Omnes, M. Defour, M. Razeghi, and B. Drevillon, J. Appl. Phys. 68, 3564 (1990).

[17] T. Yasuda, D. E. Aspnes, D. R. Lee, C. H. Bjorkman, and G. Lucovsky, J. Vac. Sci. Technol. A 12, 1152 (1994).

[18] O. Hunderi, J. Zettler, and K. Haberland, Thin Solid Films 472, 261 (2005).

[19] T. Nakayama, Phys. Status Solidi B 202, 741 (1997).

[20] J. D. E. McIntyre and D. E. Aspnes, Surf. Sci. 24, 417 (1971).

[21] T. Yasuda, Thin Solid Films 313-314, 544 (1998).

[22] R. Dovesi, V. R. Saunders, C. Roetti, R. Orlando, C. M. Zicovich-Wilson, F. Pascale, B. Civalleri, K. Doll, N. M. Harrison, I. Bush et al., Crystal09 User's Manual (University of Torino, Torino, 2009).

[23] C. H. Patterson, Mol. Phys. 108, 3181 (2010).

[24] R. Pandey, M. Causa, N. M. Harrison, and M. Seel, J. Phys. Condens. Matter 8, 3993 (1996).

[25] C. M. Zicovich-Wilson, A. Bert, C. Roetti, R. Dovesi, and V. R. Saunders, J. Chem. Phys. 116, 1120 (2002).

[26] F. Pascale, M. Catti, A. Damin, R. Orlando, V. Saunders, and R. Dovesi, J. Phys. Chem. B 109, 18522 (2005).

[27] M. F. Peintinger, D. V. Oliveira, and T. Bredow, J. Comput. Chem. 34, 451 (2013).

[28] T. Leininger, A. Berning, A. Nicklass, H. Stoll, H.-J. Werner, and H.-J. Flad, Chem. Phys. 217, 19 (1997).

[29] A. Bergner, M. Dolg, W. Kuechle, H. Stoll, and H. Preuss, Mol. Phys. 80, 1431 (1993).

[30] H. Monkhorst and J. D. Pack, Phys. Rev. B 13, 5188 (1976).

[31] A. D. Becke, J. Chem. Phys. 98, 5648 (1993).

[32] W. G. Schmidt, E. L. Briggs, J. Bernholc, and F. Bechstedt, Phys. Rev. B 59, 2234 (1999).

[33] C. Hogan, R. DelSole, and G. Onida, Phys. Rev. B 68, 035405 (2003).

[34] B. S. Mendoza, F. Nastos, N. Arzate, and J. E. Sipe, Phys. Rev. B 74, 075318 (2006).

[35] W.-K. Wang and M.-C. Jiang, Jpn. J. Appl. Phys. 55, 095503 (2016).

[36] S. Liu, M. Peng, C. Hou, Y. He, M. Li, and X. Zheng, Nanoscale Res. Lett. 12, 279 (2017).

[37] N. Pavlenko, T. Kopp, E. Y. Tsymbal, J. Mannhart, and G. A. Sawatzky, Phys. Rev. B 86, 064431 (2012).

[38] V. Vonk, J. Huijben, D. Kukuruznyak, A. Stierle, H. Hilgenkamp, A. Brinkman, and S. Harkema, Phys. Rev. B 85, 045401 (2012).

[39] B. Förg, C. Richter, and J. Mannhart, Appl. Phys. Lett. 100, 053506 (2012).

[40] S. Y. Park and A. J. Millis, Phys. Rev. B 87, 205145 (2013).

[41] T. Asmara, A. Annadi, I. Santoso, P. Gogoi, A. Kotlov, H. Omer, M. Motapothula, M. Breese, and M. Rhausen, T. Venkatesan et al., Nat. Commun. 5, 3663 (2014). 